\title{
A multi-stable memristor and its application in a neural network
}

\author{
Hairong Lin, Chunhua Wang, Qinghui Hong and Yichuang Sun, Senior Member, IEEE
}

\begin{abstract}
Nowadays, there is a lot of study on memristorbased systems with multistability. However, there is no study on memristor with multistability. This brief constructs a mathematical memristor model with multistability. The origin of the multi-stable dynamics is revealed using standard nonlinear theory as well as circuit and system theory. Moreover, the multi-stable memristor is applied to simulate a synaptic connection in a Hopfield neural network. The memristive neural network successfully generates infinitely many coexisting chaotic attractors unobserved in previous Hopfield-type neural networks. The results are also confirmed in analog circuits based on commercially available electronic elements.
\end{abstract}

Index Terms-Multistability, memristor, circuit theory, neural networks, nonlinear circuits.

\section{INTRODUCTION}

$\mathbf{M}$ EMRISTOR with nonlinearity and nonvolatility is considered as the fourth basic electronic element [1]. It has become a research hotspot in academia and industry since the discovery of the Hewlett-Packard (HP) memristor nano-device in 2008 [2]. Driven by engineering applications, scholars and technologists have devoted great enthusiasm to memristor models and their applications. Up to now, numerous different types of memristor models including theoretical models, circuit models and material models have been developed [3-6].

Recently, the study of pure mathematical memristor models has been of interest. The mathematical memristors provide several prominent features like excellent memristive characteristic, strong nonlinearity, low cost, and easy circuit implementation as compared with other memristors. Furthermore, the mathematical memristors can be widely applied in many fields due to their simple and flexible mathematical expression. Chua proposed many important theories on mathematical memristors and classified them into ideal memristor, generic memristor and extended memristor [7,8], which provides a sufficient theoretical basis for further research of mathematical memristors. Bao et al. created some simple mathematical memristors by using different nonlinear functions such as absolute value function [9], quadratic function [10] and hyperbolic tangent function [11], and these memristors have been widely used

Manuscript received April 8, 2020. This work is supported by The Major Research Project of the National Natural Science Foundation of China (91964108), The National Natural Science Foundation of China (61971185), The Open Fund Project of Key Laboratory in Hunan Universities (18K010). (Corresponding auther: Chunhua Wang.)

Hairong Lin, Chunhua Wang and Qinghui Hong are with the College of Computer Science and Electronic Engineering, Hunan University, Changsha, 410082, China (e-mail: haironglin66@126.com; wch1227164@hnu.edu.cn; hongqinghui@hnu.edu.cn).

Yichuang Sun is with the School of Engineering and Computer Science, University of Hertfordshire, Hatfield AL10 9AB, U.K.(y.sun@ herts.ac.uk). in various chaotic circuits [12] and artificial neural networks [13]. Wang et al. designed a generic memristor with local activity, and proposed a simplest chaotic circuit by employing this memristor [14]. In addition, Ascoli et al. constructed an extended bistable memristor with two coexisting pinched hysteresis loops under different initial states [15]. Meanwhile, many important achievements based on the mathematical memristors have been made constantly, especially the investigation of memristor-based multi-stable systems [16-19]. Although many memristive multi-stable systems have been developed by using existing memristors, the generation of their multi-stable behavior is stochastic and uncontrolled. That is, the memristor-based multi-stable systems can only be achieved under some special initial conditions. In particular, it is difficult to observe the clear multi-stable behavior in physical experiments since these special initial conditions are basically impossible to obtain in hardware circuits. It is therefore of great significance to provide a memristor model that accurately and effectively maps multi-stable behavior in memristive multi-stable systems.

In this brief, we propose a memristor with multistability. To the best of our knowledge, there is no multi-stable memristor until now. The key mechanism underlying the emergence of multistability in this memristor are elucidated. The multi-stable memristor can exhibit infinitely many coexisting pinched hysteresis loops under different tunable initial states. Compared with the other memristors, the multi-stable memristor is more suitable for the development of memristive multi-stable systems due to its multistability and initial condition tunability. Besides, we establish a memristive Hopfield neural network by utilizing the multi-stable memristor as a memristor synapse. The memristive neural network generates infinitely many coexisting chaotic attractors that enjoy the same structure, which is different from the multistability in the existed memristive systems. And their generation is attributed to the duplication of the original attractor, which is predictable and tunable.

\section{Multi-Stable Memristor MOdel}

According to the memristor theory [8], a voltage-controlled generic memristor model can be defined as

State-dependent Ohm's law:

$$
i=G(x) v,
$$

State equation:

$$
d x / d t=g(x, v),
$$

where $G(x)$ is memductance, and $v, i, x$ denote voltage, current, and memristor state, respectively. Now, we propose 
a generic memristor model based on Equations (1) and (2), namely

$$
\begin{gathered}
i=G(x) v=x v, \\
d x / d t=g(x, v)=\sin (x)+v .
\end{gathered}
$$

Consider an external sinusoidal stimulus imposed on the memristor, namely,

$$
v=A \sin (F t),
$$

where $A$ and $F$ denote amplitude and frequency, respectively. Consequently, the solution of the state equation (4) can be calculated by

$$
\begin{aligned}
& x=\int_{-\infty}^{t}(\sin (x)+v) d \tau \\
& =x_{0}-\cos (x)+(A / F)(1-\cos (F t))
\end{aligned}
$$

where $x_{0}$ is the initial state of the memristor. Substituting Equations (5) and (6) into Equation (3), then have

$$
\begin{aligned}
& i=\left(x_{0}-\cos (x)+(A / F)(1-\cos (F t))\right)(A \sin (F t)) \\
& =\left(x_{0}-\cos (x)+A / F\right)(A \sin (F t)) \\
& -\left(A^{2} / 2 F\right) \sin (2 F t)
\end{aligned}
$$

Obviously, the resulting output is composed of a fundamental component and a harmonic component, which depends on both initial state and transient state of the memristor and both amplitude and frequency of the applied input. More deep analysis is given in the next text, where all numerical simulations are completed by the Matlab 2017a tool with the ODE45 algorithm.

\section{A. Volt-ampere Characteristics}

Equations (3) and (4) are studied under different frequencies and amplitudes, when the stimulus $v=A \sin (F t)$ is selected as the driving source. When $x_{0}=0, F=0.8$ are fixed with different values of the amplitude $A$, and $x_{0}=0, A=1.4$ are fixed with different values of the frequency $F$, the pinched hysteresis loops of the memristor are numerically simulated and plotted in Fig.1(a) and Fig.1(b), respectively. Obviously, in Fig.1, six pinched hysteresis loops pass through the origin in the voltagecurrent $(v-i)$ plane when driven by sinusoidal signal with different amplitudes and frequencies. And in Fig.1(b), as the excitation frequency increases from 0.8 to 4.8 , the hysteresis lobe area is gradually decreased. Furthermore, it is evident that when the frequency increases to infinity the pinched hysteresis loop will tend to a single-valued function. Consequently, the proposed model is a memristor device because it exhibits three fingerprints of memristor [7].
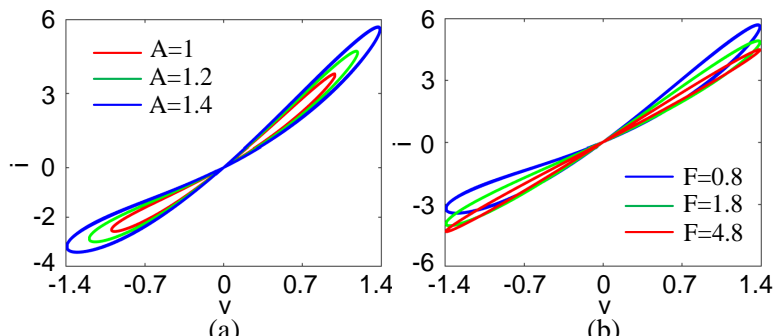

Fig. 1. Pinched hysteresis loops of the multi-stable memristor under $v=A \sin (F t)$ with $x_{0}=0$. (a) $F=0.8$ and different $A$. (b) $A=1.4$ and different $F$.

\section{B. Multistability Analysis}

Here, power-off plot (POP) is used to disclose the multistability of the memristor. Usually, POP is a curve in the $d x / d t(v=0)$ versus $x$ plane [8]. It should be stressed that each intersection of POP with the $x$-axis is defined as an equilibrium point of the memristor due to $d x / d t=0$. Let $v=0$, the memristor state equation (4) reduces to

$$
d x / d t=g(x, 0)=\sin (x) .
$$

The dynamic route of the dynamical equation (8), namely POP, is shown in Fig.2. In Fig.2, when $d x / d t=0$, there are infinitely many intersections, namely equilibrium points located at $x=k \pi(k \in Z)$. According to the judgment method of [8], the equilibrium points marked by polka dots are asymptotically stable, while the equilibrium points marked by square dots are unstable. Evidently, when $k$ is even number, the equilibrium point $S_{k}$ is unstable, and the equilibrium point $S_{k+1}$ is stable.
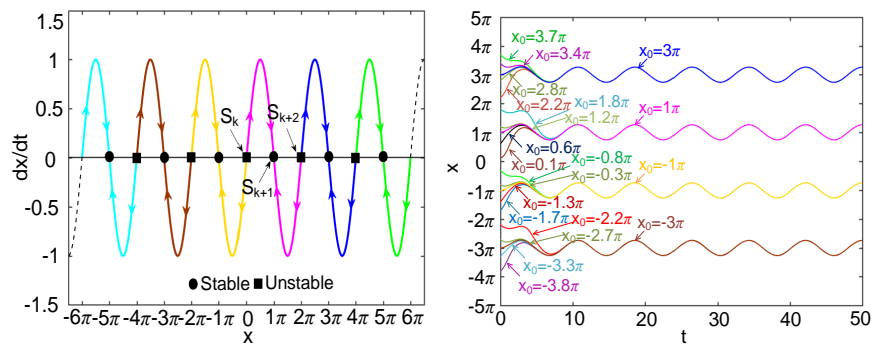

Fig. 2. POP of the multi-stable mem- Fig. 3. Time evolution of the multiristor, where $k$ denotes even number. stable memristor state toward different stable states for different initial states.

Thus, for initial state $x_{0} \in(k \pi,(k+2) \pi)$, the memristor state tends to the stable equilibrium point $x\left(S_{k+1}\right)$, namely

$$
\left.x=x\left(S_{k+1}\right)=(k+1) \pi \text {, if } \quad k \pi<x_{0}<(k+2) \pi\right) .
$$

Equation (9) directly illustrates two important results: (i) for different initial states with different $k$, the dynamical trajectory of the multi-stable memristor will tend different stable equilibrium points, which means multistability. (ii) for different initial states with the same $k$, the dynamical trajectory of the multi-stable memristor will tend the same stable equilibrium point, which means tunability. Numerical simulation results from Equations (3) and (4) under $v=A \sin (F t)$ with $A=1$ and $F=0.8$ verify the above two conclusions, as shown in Fig.3. For example, when $k=-4,-2,0$ and 2 , for initial states in four ranges $(-4 \pi,-2 \pi),(-2 \pi, 0),(0,2 \pi)$ and $(2 \pi, 4 \pi)$, the dynamical trajectory $x$ tends to four stable equilibrium points $x=-3 \pi$, $1 \pi, 1 \pi$ and $3 \pi$, respectively. While for different initial state in the same range such as $x_{0}=2.2 \pi, 2.8 \pi, 3 \pi, 3.4 \pi, 3.7 \pi$, the dynamical trajectory tends to the same stable equilibrium point $x=3 \pi$. Therefore, infinitely many stable equilibrium states exist in the multi-stable memristor for infinite adjustable initial state ranges. These stable states generate the following infinitely many corresponding memductances

$$
G\left(x\left(S_{(k+1)}\right)\right)=(k+1) \pi \text {, if } \quad k \pi<x_{0}<(k+2) \pi .
$$

where $k$ is even number. In other words, for different $k$, the memristor gives rise to different memductances. As shown in Fig.4(a), under eight initial states $x_{0}=7 \pi, 5 \pi, 3 \pi, 1 \pi,-1 \pi,-3 \pi$, 
$-5 \pi$ and $-7 \pi$, corresponding eight different memductances can be induced on the multi-stable memristor under $v=A \sin (F t)$ with $A=1, F=0.8$. Obviously, such multi-stable memristor is also non-volatile because the infinitely many memductance $G$ can be used to represent countless memory states. Meanwhile, the multi-stable memristor produces eight different pinched hysteresis loops on $v-i$ plane, as shown in Fig.4(b). It can thus be inferred that the multi-stable memristor can yield coexisting infinitely many pinched hysteresis loops for different initial state ranges $x_{0} \in(k \pi,(k+2) \pi)$, where $k$ is any even number.

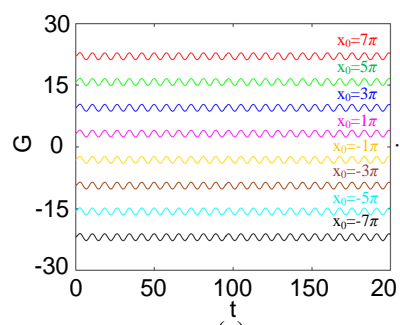

(a)

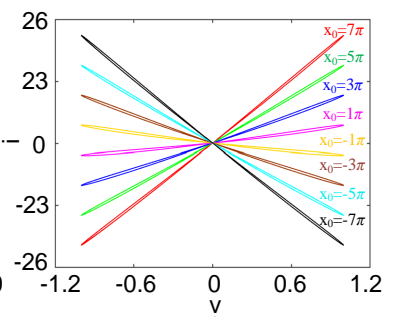

(b)
Fig. 4. Numerical simulation results of the multi-stable memristor in (3)-(4) under different initial states $x_{0}=-7 \pi,-5 \pi,-3 \pi,-1 \pi, 1 \pi, 3 \pi, 5 \pi$ and $7 \pi$. (a) Memductances. (b) coexisting Pinched hysteresis loops.

Let us gain a deeper insight into this aspect by using the circuit theoretic technique direct current voltage-current (DC $\left.V_{m}-I_{m}\right)$ loci $[8,15]$. The DC $V_{m}-I_{m}$ loci of the multi-stable memristor can be derived by considering Equations (3) and (4) with the input DC $V_{m}$ value varying from $-1 \mathrm{~V}$ to $1 \mathrm{~V}$ and the memristor state $x$ value varying within $(-8 \pi, 8 \pi)$, as shown in Fig.5. The DC $V_{m}-I_{m}$ loci of the memristor are composed of eight stable branches, which implies that the memristor exhibits multiple stable state behavior under direct current stimulus. It is obvious that for different memristor state ranges $x \in(k \pi,(k+2) \pi)$ where $k$ is even number, the multi-stable memristor will generate different stable branches. Indeed, we can verify that more coexisting stable branches will be observed by selecting more variable intervals, which strongly illustrates the multistability of the multi-stable memristor. Furthermore, in Fig.5, for the branches of state $x<0$, their slope at origin are negative, which means that the multi-stable memristor is also locally active.
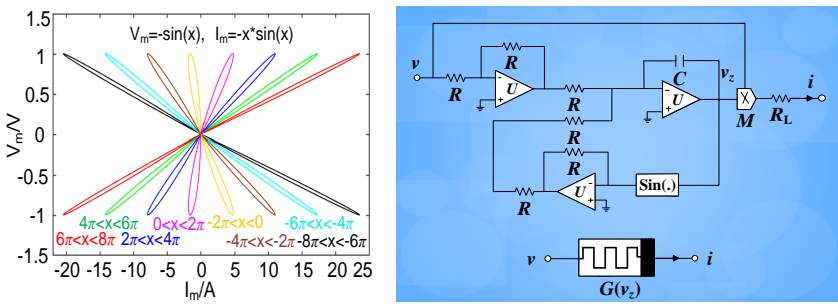

Fig. 5. DC Vm-Im loci of the Fig. 6. Circuit configuration of the multimulti-stable memristor. stable memristor.

\section{Circuit Implementation}

We design and implement the hardware circuit of the multi-stable memristor by adopting resistors, capacitors, operational amplifiers TL082CP, analog multipliers AD633JN and trigonometric function converters AD639AD all powered by $\pm 18 \mathrm{~V}$ symmetric voltages. Fig.6 gives the circuit schematic diagram of the multi-stable memristor. The memristor state $x$ is simulated by the output voltage of integrator, namely $v_{z}$. According to the basic electrical circuit's law, the circuital equations of the multi-stable memristor can be rewritten as

$$
\begin{gathered}
i=G\left(v_{z}\right) v=\frac{v_{z}}{R_{L}} v, \\
R C \frac{d v_{z}}{d t}=\sin \left(v_{z}\right)+v .
\end{gathered}
$$

Let $C=10 \mathrm{nF}, \quad R=R_{L}=10 \mathrm{k} \Omega, v=H \sin (W t)$ with $H=1$ and $W=8000$, coexisting six pinched hysteresis loops can be obtained from the multi-stable memristor circuit in PSIM simulation with respect to the initial values of the voltages of capacitor $C$ fixed as $-5 \pi \mathrm{V},-3 \pi \mathrm{V},-1 \pi \mathrm{V}, 1 \pi \mathrm{V}, 3 \pi \mathrm{V}, 5 \pi \mathrm{V}$, as shown in Fig.7a. Moreover, the corresponding simulated memristor states $v_{z}$ are shown as in Fig.7b. It is clear that the memristor state tends to different stable state under different initial capacitor voltages in the memristor circuit, which further verifies the above theoretical analysis and numerical simulation results.

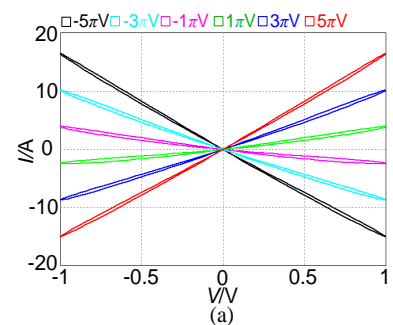

(a)

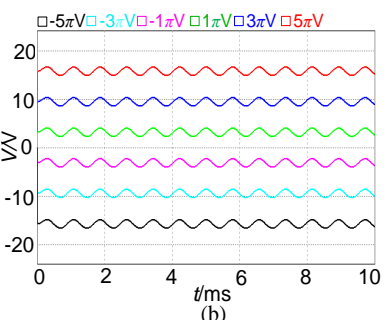

Fig. 7. Circuit simulation results of the multi-stable memristor under different initial capacitor voltages $-5 \pi \mathrm{V},-3 \pi \mathrm{V},-1 \pi \mathrm{V}, 1 \pi \mathrm{V}, 3 \pi \mathrm{V}, 5 \pi \mathrm{V}$. (a) Six coexisting pinched hysteresis loops. (b) memristor state $v_{z}$.

\section{ApPlicAtion In A HOPFIELD NEURAL NETWORK}

Hopfield neural network is often used to imitate the dynamical behavior of brain activities [20]. The neural network with $n$ neurons can be described by

$$
C_{i} \dot{x}_{i}=-x_{i} / R_{i}+\sum_{j=1}^{n} w_{i j} \tanh \left(x_{i}\right)+I_{i} \quad\left(i, j \in N^{*}\right),
$$

where $C_{i}, R_{i}$, and $x_{i}$ are the membrane capacitance, membrane resistance, and membrane voltage between the outside and inside of the neuron $i$, respectively. $\tanh \left(x_{i}\right)$ is the neuron activation function, and $I_{i}$ is external bias current. $w_{i j}$ is defined as synaptic weight describing the strength of connection from neuron $j$ to neuron $i$. From the electronic point of view, $w_{i j}$ is the admittance of the resistor connecting the neuron $j$ and neuron $i$. According to the theory of memristors, both memductance and $w_{i j}$ are in the units of Siemens, which means that the connection resistor can be replaced by a memristor. As a result, the synaptic weight can be described by the memductance of a memristor. Under this strategy, some memristor-based Hopfield neural network were, thereby, presented and complex dynamical behaviors of hyperchaos, chimera and coexisting attractors were discovered [21-23]. Here we propose a memristive Hopfield neural network with four neurons based on this strategy, as shown in Fig.8. It is 


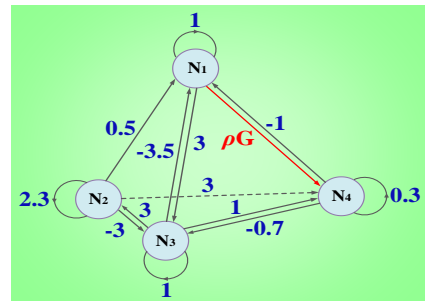

Fig. 8. Connection topology of the memristive neural network with four neurons.

noting that the proposed multi-stable memristor plays the role of the synaptic weight $w_{41}$ in this neural network. Combining Equations (3), (4) and (13), let $C_{i}=1, R_{i}=1$ and $I_{i}=0$, the dynamical equations of the memristive neural network in Fig.8 are derived as

$$
\left\{\begin{array}{l}
\dot{x_{1}}=-x_{1}+\tanh \left(x_{1}\right)+0.5 \tanh \left(x_{2}\right)-3.5 \tanh \left(x_{3}\right)-\tanh \left(x_{4}\right) \\
\dot{x_{2}}=-x_{2}+2.3 \tanh \left(x_{2}\right)+3 \tanh \left(x_{3}\right) \\
\dot{x_{3}}=-x_{3}+3 \tanh \left(x_{1}\right)-3 \tanh \left(x_{2}\right)+\tanh \left(x_{3}\right)-0.7 \tanh \left(x_{4}\right) \\
\dot{x_{4}}=-x_{4}+\rho G \tanh \left(x_{1}\right)+3 \tanh \left(x_{2}\right)+\tanh \left(x_{3}\right)+0.3 \tanh \left(x_{4}\right) \\
\dot{z}=\sin (z)+\tanh \left(x_{1}\right)
\end{array}\right.
$$

where memductance $G=z$ stands for the synaptic weight $w_{41}$ connecting the neuron 1 and neuron 4 , and system parameter $\rho$ is the coupling strength of the memristor. By letting the right of neural network (14) equal to zero, we find that the memristive neural network has infinitely discrete equilibria which can be expressed as

$$
E=\left\{\left(x_{1}^{*}, x_{2}^{*}, x_{3}^{*}, x_{4}^{*}, z^{*}\right) \mid x_{1}^{*}=x_{2}^{*}=x_{3}^{*}=x_{4}^{*}=0, z^{*}=k \pi\right\},
$$

where $k \in Z$. We can see that the memristive neural network generates infinitely many equilibria along the axis- $z$ by changing phase space. Obviously, the multi-stable memristor synapse plays a key role for the generation of the infinite equilibria. Unique behavior in neural network (14) is investigated in the following content.
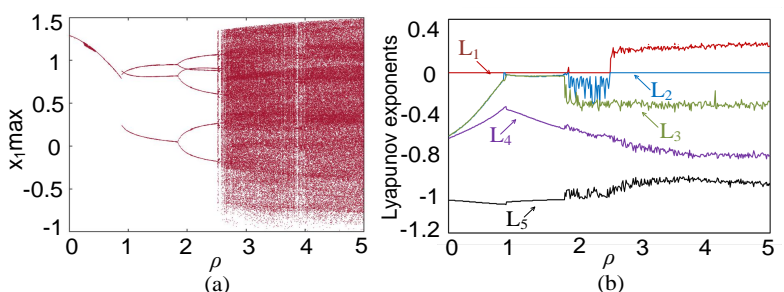

Fig. 9. Bifurcation diagram and Lyapunov exponents of the memristive neural network with $\rho \in(0,5)$ and initial states $(1,1,1,1,1)$. (a) Bifrucation diagram. (b) Lyapunov exponents.

Supposing that the initial states $\left(x_{10}, x_{20}, x_{30}, x_{40}, z_{0}\right)=(1$, $1,1,1,1)$, we can plot the bifurcation diagram and Lyapunov exponents of the memristive neural network with respect to the parameter $\rho \in(0,5)$ as shown in Fig. 9. It can be seen from Fig.9 that the memristive neural network appears forward period doubling bifurcation with the increase of $\rho$. And when $\rho$ increases to 2.8 , the neural network enters stable chaotic states. The phase portraits of the attractors of the neural network with different values of $\rho$ are given to illustrate its dynamical evolution with the coupling strength of the memristor, as shown in Fig.10. It is evident that the memristive neural network successively generates periodic attractor with different period and chaotic attractor with the increase of $\rho$ under initial states $(1,1,1,1,1)$.

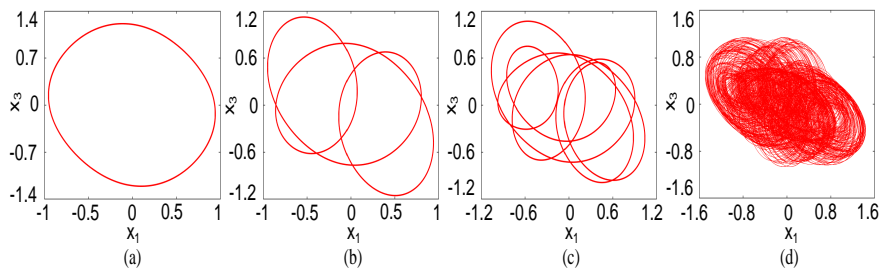

Fig. 10. Numerically simulated phase portraits for different $\rho$. (a) Period-1 attractor at $\rho=0.7$. (b) Period-3 attractor at $\rho=1.5$. (c) Period-6 attractor at $\rho=2.2$. (d) Chaotic attractor at $\rho=4$.

It is more interesting that the memristive neural network can generate infinitely many coexisting chaotic attractors under different initial states for fixed coupling strength of the multistable memristor. Let $\rho=4$, infinitely many attractors can be observed from the following initial states $\left(x_{10}, x_{20}, x_{30}, x_{40}\right.$, $\left.z_{0}\right)=(1,1,1,1, m \pi)$ where $m=1,3,5 \ldots$ For instance, by selecting eight initial states $(1,1,1,1, \pi),(1,1,1,1,3 \pi)$, $(1,1,1,1,5 \pi),(1,1,1,1,7 \pi),(1,1,1,1,9 \pi),(1,1,1,1$, $11 \pi),(1,1,1,1,13 \pi)$ and $(1,1,1,1,15 \pi)$, eight coexisting chaotic attractors can be generated from the memristive neural network, as shown in Fig.11. And we can confirm that more coexisting chaotic attractors will be found via selecting more initial states.

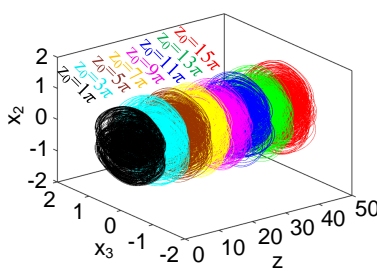

(a)

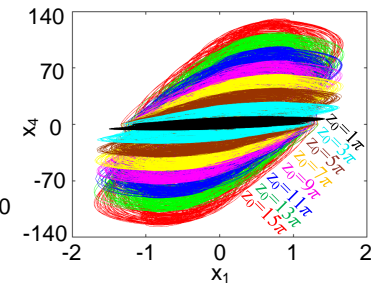

(b)
Fig. 11. Eight coexisting chaotic attractors on different phase space with different initial conditions and $\rho=4$. (a) $z-x_{3}-x_{2}$. (b) $x_{1}-x_{4}$.

Furthermore, when $x_{10}, x_{20}, x_{30}, x_{40}$ are kept as 1 , the bifurcation diagram and Lyapunov exponents of the memristive neural network with respect to $z_{0} \in[1 \pi, 19 \pi]$ are plotted for further illustrating the coexistence of infinitely many chaotic attractors in Fig.12. Fig.12 directly shows two important phenomena: (i) memristive neural network (14) generates infinitely many chaotic attractors with different position with the increase of $z_{0}$; (ii) each attractor has an independent initial state range $z_{0} \in(k \pi,(k+2) \pi)$ where $k$ is even number. Clearly, the position and initial state of each attractor are tunable, which means that the multi-stable dynamics in neural network (14) is controllable.

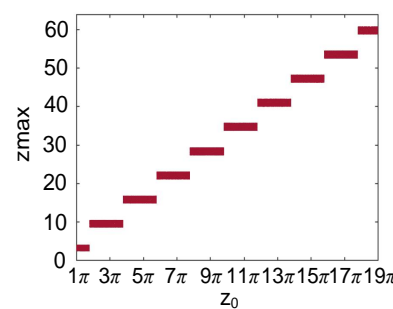

(a)

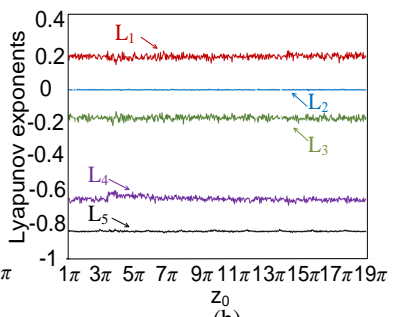

(b)
Fig. 12. Bifurcation diagram and Lyapunov exponents of the memristive neural network with $z_{0} \in(1 \pi, 19 \pi)$ and $\rho=4$. (a)Bifurcation diagram. (b)Lyapunov exponents. 


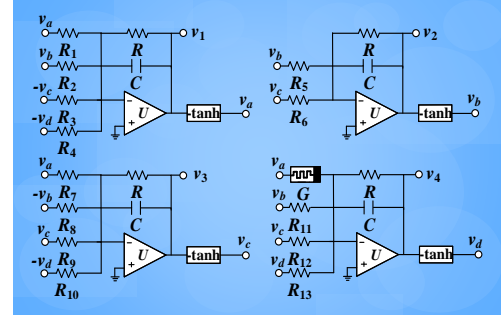

Fig. 13. Circuit configuration of the memristive neural network.

To further confirm the observed coexisting attractors, we design the circuit of the memristive neural network, as shown in Fig.13. Four membrane potentials $x_{1}, x_{2}, x_{3}, x_{4}$ are emulated by the four output voltages $v_{1}, v_{2}, v_{3}, v_{4}$, respectively. The synaptic weight $w_{41}$ is simulated by the memductance $G$ of the multi-stable memristor, other fixed synaptic weights are replaced by using resistors $R_{1}-R_{13}$, and the neural active function tanh is realized using a hyperbolic tangent function circuit designed by [11]. The circuit equations are given by

$$
\left\{\begin{array}{l}
C \frac{d v_{1}}{d t}=-\frac{v_{1}}{R}+\frac{\tanh \left(v_{1}\right)}{R_{1}}+\frac{\tanh \left(v_{2}\right)}{R_{2}}-\frac{\tanh \left(v_{3}\right)}{R_{3}}-\frac{\tanh \left(v_{4}\right)}{R_{4}} \\
C \frac{d v_{2}}{d t}=-\frac{v_{2}}{R}+\frac{\tanh \left(v_{2}\right)}{R_{5}}+\frac{\tanh \left(v_{3}\right)}{R_{6}} \\
C \frac{d v_{3}}{d t}=-\frac{v_{3}}{R}+\frac{\tanh \left(v_{1}\right)}{R_{7}}-\frac{\tanh \left(v_{2}\right)}{R_{8}}+\frac{\tanh \left(v_{3}\right)}{R_{9}}-\frac{\tanh \left(v_{4}\right)}{R_{10}} \\
C \frac{d v_{4}}{d t}=-\frac{v_{4}}{R}+\frac{v_{z} \tanh \left(v_{1}\right)}{R_{L}}+\frac{\tanh \left(v_{2}\right)}{R_{11}}+\frac{\tanh \left(v_{3}\right)}{R_{12}}+\frac{\tanh \left(v_{4}\right)}{R_{13}} \\
C \frac{d v_{z}}{d t}=\frac{\sin \left(v_{z}\right)}{R}+\frac{\tanh \left(v_{1}\right)}{R}
\end{array}\right.
$$

Considering $C=10 \mathrm{nF}, R=10 \mathrm{k} \Omega$, we have the resistances $R_{L}=R / \rho, R_{1}=R_{4}=R_{9}=R_{12}=R / 1=10 \mathrm{k} \Omega, R_{2}=R / 0.5=20 \mathrm{k} \Omega, R_{3}$ $=R / 3.5=2.85 \mathrm{k} \Omega, R_{5}=R / 2.3=4.35 \mathrm{k} \Omega, R_{6}=R_{7}=R_{8}=R_{11}=R / 3=$ $3.33 \mathrm{k} \Omega, R_{10}=R / 0.7=14.28 \mathrm{k} \Omega, R 13=R / 0.3=33.33 \mathrm{k} \Omega$. When $R_{L}=2.5 \mathrm{k} \Omega$, the initial values of the four capacitor voltages fixed as $(1 \mathrm{~V}, 1 \mathrm{~V}, 1 \mathrm{~V}, 1 \mathrm{~V}, 1 \pi \mathrm{V}),(1 \mathrm{~V}, 1 \mathrm{~V}, 1 \mathrm{~V}, 1 \mathrm{~V}, 3 \pi \mathrm{V}),(1 \mathrm{~V}, 1 \mathrm{~V}$, $1 \mathrm{~V}, 1 \mathrm{~V}, 5 \pi \mathrm{V}),(1 \mathrm{~V}, 1 \mathrm{~V}, 1 \mathrm{~V}, 1 \mathrm{~V}, 7 \pi \mathrm{V}),(1 \mathrm{~V}, 1 \mathrm{~V}, 1 \mathrm{~V}, 1 \mathrm{~V}, 9 \pi \mathrm{V})$, $(1 \mathrm{~V}, 1 \mathrm{~V}, 1 \mathrm{~V}, 1 \mathrm{~V}, 11 \pi \mathrm{V})$, six coexisting chaotic attractors are obtained from the neural network circuit in PSIM simulations, as shown in Fig. 14. Evidently, the results well verified the numerical simulation results.
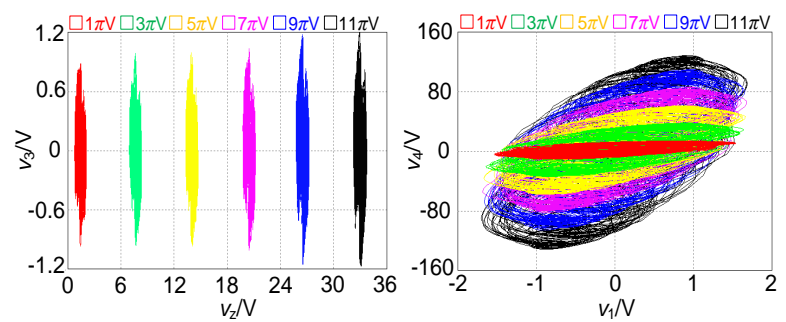

Fig. 14. Six coexisting chaotic attractors obtained from the memristive neural network circuit in PSIM simulation. (a) $v_{z}-v_{3}$. (b) $v_{1}-v_{4}$.

\section{CONCLUSION}

This brief presented a multi-stable memristor model. The memristor can exhibit infinitely many coexisting pinched hysteresis loops under different initial state ranges. Various characteristics of this memristor has been demonstrated via theoretical analysis, numerical simulation and circuit implementation. Besides, the multi-stable memristor was applied to construct a memristive neural network which can generate controllable multi-stable behavior with infinitely many coexisting chaotic attractors. Such multi-stable memristor has more important consequences in memristor-based multi-stable systems due to its multistability and initial state tunability.

\section{REFERENCES}

[1] L. O. Chua, "Memristor-the missing circuit element," IEEE Trans.Circuit Theory., vol. 18, no. 5, pp. 507-519, Sep. 1971.

[2] D. B. Strukov, G. S. Snider, D. R. Stewart and R. S. Williams, "The missing memristor found," Nature., vol. 453, no. 7191, pp. 80-83, 2008.

[3] J. Zha, H. Huang and Y. Liu, "A novel window function for memristor model with application in programming analog circuits," IEEE Trans. Cir. Sys.-II: Brief Papers., vol. 63, no. 5, pp. 423-427, 2016.

[4] Y. Zhang, X. Wang, Y. Li and E. G. Friedman, "Memristive model for synaptic circuits," IEEE Trans. Cir. Sys.-II: Brief Papers., vol. 64, no. 7, pp. 767-771, 2017.

[5] Y. Tan, C. Wang, "A simple locally active memristor and its application in HR neurons," Chaos., vol. 30, no. 5, pp. 053118, 2020.

[6] X. Zhang, W. Wang, Q. Liu, et al., "An artificial neuron based on a threshold wwitching memristor," IEEE Electron Device Lett., vol. 39, no. 2, pp. 308-311, 2018.

[7] L. O. Chua, "If it's pinched it's a memristor," Semicond. Sci. Technol., vol. 29, no. 10, pp. 104001, 2014.

[8] L. O. Chua, "Everything you wish to know about memristors but are afraid to ask," Radioengineering., vol. 24, no. 2, pp. 319-368, 2015.

[9] H. Bao, N. Wang, B. Bao, et al, "Initial condition-dependent dynamics and transient period in memristor-based hypogenetic Jerk system with four line equilibria," Commun. Nonlinear Sci. Numer. Simul., vol. 57, pp. 264-275, 2018.

[10] M. Chen, M. Sun, H. Bao, Y. Hu and B. Bao, "Flux-charge analysis of two-memristor-based Chuas circuit: dimensionality decreasing model for detecting extreme multistability," IEEE Trans. Ind. Electron., vol. 67, no. 3, pp. 2197-2206, 2020

[11] H. Bao, A. Hu, W. Liu and B. Bao, "Hidden bursting firings and bifurcation mechanisms in memristive neuron model with threshold electromagnetic induction," IEEE Trans. Neural Netw. Learn. Syst., vol. 31 , no. 2, pp. 502-511, 2020.

[12] F. Corinto, M. Forti, "Memristor circuits: bifurcations without parameters," IEEE Trans. Circuits Syst. I-Regul. Pap., vol. 64, no. 6, pp. 1540-1551, 2017.

[13] H. Lin and C. Wang, "Influences of electromagnetic radiation distribution on chaotic dynamics of a neural network," Appl. Math. Comput., vol. 369 , p. 124840, 2020.

[14] P. Jin, G. Wang, H. Iu and T. Fernando, "A locally active memristor and its application in a chaotic circuit," IEEE Trans. Cir. Sys.-II: Brief Papers., vol. 65, no. 2, pp. 246-250, 2018.

[15] A. Ascoli, R. Tetzlaff and L. O. Chua, "The first ever real bistable memristors - part I:theoretical insights on local fading memory," IEEE Trans. Cir. Sys.-II: Brief Papers., vol. 63, no. 12, pp. 1091-1095, 2016.

[16] H. Lin, C. Wang, Y. Sun and W. Yao, "Firing multistability in a locally active memristive neuron model," Nonlinear Dyn., 2020. doi.org/10.1007/s11071-020-05687-3.

[17] M. D. Marco, M. Forti and L. Pancioni, "Convergence and multistability of nonsymmetric cellular neural networks with memristors," IEEE Trans. Syst., Man, Cybern., Syst., vol. 47, no. 10, pp. 2970-2983, 2017.

[18] M. Chen, X. Ren, H. Wu, et al, "Periodically varied initial offset boosting behaviors in a memristive system with cosine memductance," Front. Inform. Technol. Elect. Eng., vol. 20, no. 12, pp. 1706-1716, 2019.

[19] H. Bao, M. Chen, H. Wu and B. Bao, "Memristor initial-boosted coexisting plane bifurcations and its extreme multi-stability reconstitution in two-memristor-based dynamical system," Sci. China-Technol. Sci., vol. 63, no. 4, pp. 603-613, 2020.

[20] J. J. Hopfield, "Neurons with graded response have collective computational properties like those of 2-state neurons," Proc. Natl. Acad. Sci. USA., vol. 81, no. 10, pp. 3088-3092, 1984.

[21] Q. Li, S. Tang, H. Zeng and T. Zhou, "On hyperchaos in a small memristive neural network," Nonlinear Dyn., vol. 78, no. 2, pp. 1087-1099, 2014.

[22] F. Parastesh, S. Jafari, H. Azarnoush, et al, "Chimera in a network of memristor-based Hopfield neural network," Eur. Phys. J.-Spec. Top., vol. 228, no. 10, pp. 2023-2033, 2019.

[23] Y. Leng, D. Yu, Y. Hu, et al, "Dynamic behaviors of hyperbolic-type memristor-based Hopfield neural network considering synaptic crosstalk," Chaos., vol. 30, no. 3, pp. 033108, 2020. 